\title{
Le cadre juridique de travail des employés de service du secteur de la restauration. La question spécifique du salaire
}

The legal framework of work for service employees in the catering sector. The specific question of wages

Louis Jolin

\section{CpenEdition}

\section{Journals}

Édition électronique

URL : http://journals.openedition.org/tourisme/2367

DOI : 10.4000/tourisme.2367

ISSN : 2492-7503

Éditeur

Éditions touristiques européennes

Référence électronique

Louis Jolin, «Le cadre juridique de travail des employés de service du secteur de la restauration. La question spécifique du salaire », Mondes du Tourisme [En ligne], 16 | 2019, mis en ligne le 01 décembre 2019, consulté le 09 avril 2020. URL : http://journals.openedition.org/tourisme/2367 ; DOI : https:// doi.org/10.4000/tourisme.2367

Ce document a été généré automatiquement le 9 avril 2020.

\section{(i) $\Theta \Theta$}

Mondes du tourisme est mis à disposition selon les termes de la licence Creative Commons Attribution - Pas d'Utilisation Commerciale - Pas de Modification 4.0 International. 


\title{
Le cadre juridique de travail des employés de service du secteur de la restauration. La question spécifique du salaire
}

\author{
The legal framework of work for service employees in the catering sector. The \\ specific question of wages
}

Louis Jolin

\section{Introduction}

La croissance touristique mondiale met une pression sur l'offre touristique qui à son tour met de la pression sur la demande en main-d'œuvre. Cette même maind'œuvre est également sollicitée par d'autres secteurs d'activité, souvent plus attrayants pour l'employé. Quelles sont les nouvelles stratégies utilisées par les entreprises touristiques pour accéder à de nouveaux bassins de travailleurs et pour retenir ces derniers ? ${ }^{1}$

1 Avant même d'aborder les stratégies à mettre en place par les entreprises touristiques, il importe de considérer le cadre juridique, qui facilite ou non le recrutement des travailleurs de service dans les restaurants. Ce cadre juridique, au Québec, est celui établi principalement par la Loi sur les normes de travail (RLRQ, c. N-1.10), modifiée en juin 2018 par la LQ 2018 c.21., mais aussi par le Code du travail (RLRQ, c. C-27) pour les salariés syndiqués ou en voie de l'être.

2 Le présent texte porte donc sur les principales dispositions touchant le salaire découlant de la loi sur les normes du travail au Québec et analyse les évolutions en cours et les modifications possibles. De manière sommaire, un parallèle sera fait avec le cadre juridique français pour mieux comprendre les spécificités de la législation québécoise en offrant un éclairage comparatif 
3 Le même exercice pourrait toucher les dispositions relatives au processus d'embauche, au contrat de travail, aux congés payés, aux vacances, à la conciliation famille-travail, à la langue de travail, aux impôts à payer...

4 Mais vu les limites imposées pour notre exposé, nous nous contenterons de la rémunération des salariés, en questionnant son impact sur la rétention ou le recrutement des employés de service dans les restaurants ou les établissements d'hébergement et, plus globalement, sur l'image même du tourisme québécois.

\section{Le paradigme de recherche}

5 Le paradigme de recherche est celui du droit comme phénomène social, «qui s'intéresse à l'origine des normes, aux facteurs sociaux qui le conditionnent, au droit comme effet et reflet de la société » (Jolin, 2012, p. 209). S'il est vrai, selon ce paradigme de recherche, que le droit ne doit pas être étudié en soi de manière exclusive - ce qui est le cas avec le positivisme juridique (Kelsen, 1962 ; Tropper, 1994) - mais comme produit des transformations extérieures, il doit aussi être abordé comme producteur de transformation dans la société (Binet, 1990 ; Cubertafond, 1999). C'est en ce sens qu'on peut considérer le cadre juridique du travail comme un élément déterminant pour accéder à de nouveaux bassins de travailleurs ou du moins pour retenir ceux qui sont en place.

6 Dans sa thèse de doctorat portant sur les systèmes juridiques de détermination des salaires, le juriste réputé en droit du travail au Québec, René Laperrière (1972, tome 1, p. 2), affirmait ce qui suit :

[...] si l'on entend donner au droit un rôle social et en faire un instrument dynamique au service de l'économie, il faut dépasser l'interprétation des lois pour confronter celles-ci au contexte socioéconomique auquel elles s'appliquent; quelle est la fonction de la loi, de quelle manière affecte-t-elle la réalité des dimensions économiques, la réalité des décisions économiques et des relations sociales et comment à son tour est-elle influencée et modelée par la dynamique de cette réalité ?

7 Faut-il maintenir certaines dispositions législatives ou réglementaires, faut-il les changer si le contexte socioéconomique évolue?

Dans un contexte de relatif plein emploi au Québec, on assiste actuellement à un réel problème de recrutement et de rétention de la main-d'œuvre dans l'industrie touristique et hôtelière ${ }^{2}$. Le tourisme national et international est en constante progression, mais on constate une forte concurrence sur les produits et les prix selon les marchés réels et potentiels. Les gouvernements sont appelés à intervenir pour stimuler l'économie touristique, tout en se souciant à la fois des consommateurs et des employés du secteur.

9 Pour ces derniers, le droit du travail est sollicité avec ses spécificités nationales car les systèmes nationaux sont différents d'un pays à l'autre (p. ex. France/Canada-Québec). 


\section{De quelques différences et ressemblances entre le droit du travail au Québec et en France}

De manière sommaire, mais significative pour notre propos, retenons les caractéristiques suivantes au Québec :

- Un salaire minimum et des conditions minimales de travail sont assurés pour tous les salariés québécois en vertu de la Loi sur les normes du travail (sauf pour ceux qui travaillent dans les entreprises dites de "compétence fédérale", autrement dit dont les objets ressortent à l'autorité législative fédérale ${ }^{3}$ ). Pour les salariés des entreprises de compétence fédérale, ces normes se retrouvent dans le Code canadien du travail, (LRC [1985], ch. L-2).

- Le Code du travail québécois régit principalement le processus de syndicalisation des travailleurs (unités d'accréditation, négociation des conventions collectives, etc.).

- Dans le domaine du tourisme, de la restauration et de l'hôtellerie, il y a peu de travailleurs syndiqués dans les PME, mais la syndicalisation est largement présente dans les grandes entreprises hôtelières et de transport, ainsi que dans quelques chaînes de restaurants ${ }^{4}$.

- Les contrats individuels de travail comme les conventions collectives doivent respecter le minimum prévu à la Loi sur les normes de travail, qui est d'ordre public, mais les contrats et conventions peuvent contenir des dispositions plus favorables.

- La négociation des conventions collectives se fait principalement au plan local par unité d'accréditation. On trouve donc peu de conventions sectorielles au Québec; il en existe cependant dans certains milieux en vertu de la Loi sur les décrets de convention collective (LRQ, c. D-2), comme les agents de sécurité, le personnel d'entretien des édifices publics à Montréal et Québec, les services automobiles, le camionnage, la coiffure en Outaouais... Les conventions sectorielles dans l'industrie de la construction sont encadrées par la Loi sur les relations de travail, la formation professionnelle et la gestion de la main-d'œuvre dans l'industrie de la construction (RLRQ, c. R-20).

- Si pour une entreprise, ou une unité d'accréditation, il y a une convention collective, elle s'applique à tous les travailleurs de l'entreprise ou de l'unité ; dans ce contexte, il n'y a plus de contrat individuel...

11 Par comparaison, toujours de manière sommaire mais significative, le système national français se caractérise par les éléments suivants :

- Le Code du travail prévoit des normes nationales, dont le $\mathrm{SMIC}^{5}$, et le processus de syndicalisation.

- La syndicalisation se fait sur une base individuelle.

- Des conventions sectorielles sont présentes dans les principaux secteurs, négociées par les centrales syndicales représentatives et des associations d'employeurs. Les dispositions s'appliquent alors aux employés du secteur, syndiqués ou non.

- Il existe principalement deux conventions sectorielles dans le domaine de l'hôtellerie et de la restauration : la Convention collective nationale des hôtels, cafés, restaurants (HCR) du 30 avril $1997^{6}$, modifiée régulièrement jusqu'à ce jour par des textes attachés et des avenants; et la Convention collective du personnel des entreprises de restauration des collectivités du 20 juin $1983^{7}$, également modifiée par des annexes et avenants.

- Les contrats individuels et les conventions locales sont possibles mais elles doivent respecter au minimum les normes nationales et la convention sectorielle.

- Il faut donc tenir compte du principe de hiérarchie des normes, modifié récemment par le gouvernement Macron pour certaines conditions de travail (p. ex., la durée maximale de travail) mais pas pour le salaire (Miné, 2017). 


\section{Le salaire des salariés ou employés de la restauration et la question des pourboires}

Au Québec :

- Pour les salariés assujettis, le salaire minimum est établi par règlement gouvernemental en vertu de la Loi sur les normes du travail, article 40. À compter du $1^{\mathrm{er}}$ mai 2020 , le taux général du salaire minimum est de 13,10 \$ de l'heure, mais pour les salariés au pourboire le salaire minimum est inférieur à 10,45 $\$$.

- Est considéré comme salarié au pourboire, en vertu de l'article 3 du Règlement sur les normes du travail (c. $\mathrm{N}-1.1, \mathrm{r}-3$ ), celui qui reçoit habituellement des pourboires et qui travaille :

1- dans un établissement qui offre contre rémunération de l'hébergement à des touristes, y compris un établissement de camping ;

2- dans un local où des boissons alcooliques sont vendues pour consommation sur place ;

3- pour une entreprise qui vend, livre ou sert des repas pour consommation à l'extérieur ;

4- dans un restaurant, sauf s'il s'agit d'un lieu où l'activité principale consiste à fournir des services de restauration à des clients qui commandent ou choisissent les produits à un comptoir de service et qui paient avant de manger.

- Le pourboire ne doit pas être confondu avec le salaire; il comprend les frais de service ajoutés à la note du client (s'il y a lieu) mais ne comprend pas les frais d'administration ajoutés à la note (s'il y a lieu) (article 50 de la loi).

- En pratique, au Québec, les pourboires ne sont généralement pas inscrits sur la note du client et laissés à sa discrétion (entre 10 et $20 \%$ de la note, généralement $15 \%$ ).

- Les pourboires doivent être déclarés par le salarié pour fins d'impôt. En vertu de l'article 42.11 de la Loi québécoise sur les impôts (RLRQ., c. I-3), un pourcentage minimal de $8 \%$ sur les ventes du salarié susceptibles d'engendrer un pourboire est attribué par l'employeur sur le bulletin de paie pour le calcul des charges sociales payées par l'employeur sur le salaire de l'employé et pour les déductions à la source. Mais il y a des exceptions (p. ex., préposé au vestiaire, conjoint de l'employeur, personne qui ne reçoit qu'une cote des pourboires) ${ }^{9}$.

- La Loi sur les normes de travail permet aux salariés au pourboire de convenir entre eux d'un partage des pourboires. L'employeur ne peut intervenir. Il appartient aux salariés au pourboire d'en convenir sur une base volontaire et seuls ces salariés sont parties à la convention: les maîtres d'hôtels, les commis-débarrasseurs, les employés de cuisine ne peuvent intervenir, mais les salariés au pourboire peuvent décider de les inclure.

- La Loi sur les normes du travail édicte aussi que les heures en attente de travail sur place doivent être payées (article 57 de la loi) et qu'un minimum de trois heures doit être rémunéré lors d'une assignation au travail sur les lieux de l'entreprise (article 58 de la loi).

- Plusieurs autres dispositions de la loi ont des incidences sur le salaire (durée normale du travail, rémunération des heures supplémentaires, congés payés, etc.).

\section{Éléments d'analyse}

De cet aperçu sommaire, on peut certes affirmer que les dispositions législatives ont un impact sur les salariés au pourboire. La distinction entre les travailleurs soumis au taux général du salaire minimum et les salariés au pourboire laisse une 
partie du salaire à l'appréciation de la clientèle mais rien n'interdit au restaurateur d'imposer directement des frais de service et des frais d'administration sur la facture si les clients en sont informés préalablement. Cependant, la pratique générale en Amérique du Nord ne va pas dans ce sens, contrairement à ce que l'on retrouve dans plusieurs pays européens (notamment la France). Les salariés au pourboire sont divisés sur cette question, certains y gagneraient certainement, d'autres y perdraient... Il y a un écart important dans les PME de la restauration entre ce que gagnent les cuisiniers et les autres employés de cuisine (qui ne reçoivent pas de pourboires) et les serveurs, à l'avantage de ces derniers. Le partage volontaire des pourboires ne concerne pas, en principe, les cuisiniers.

Dans plusieurs restaurants, on retrouve un climat de tension sur cette question et un sentiment d'injustice (on est loin de l'équité salariale !). Bien sûr, des conventions collectives dans le secteur de la grande hôtellerie (p. ex. Le Reine Elizabeth à Montréal, qui fait maintenant partie du groupe Accord) accordent de bien meilleures conditions à l'ensemble du personnel, mais cela ne concerne pas les nombreux autres établissements. Il n'y a pas de convention sectorielle au Québec dans le secteur de la restauration, des cafés et des bars...

La pratique de laisser le pourboire (ou frais de service) à l'appréciation seule de la clientèle ne va pas dans le sens de la valorisation du travail des serveurs et nuit à l'image du tourisme québécois auprès de la clientèle hors Amérique du Nord. Elle peut donner lieu à du profilage racial et à de la discrimination, car il arrive que le restaurateur ajoute, dans certaines situations, les frais de service directement sur la facture sans en avoir informé préalablement le client ${ }^{10}$. Cette pratique contrevient en quelque sorte à la Loi sur la protection du consommateur (RLRQ, c. P-40.1) car les prix affichés dans les menus ne correspondent pas aux prix réels. Avec les taxes à la consommation et les pourboires ou frais de services, il y a un écart d'environ $30 \%$.

Peut-on faire autrement? Des modifications législatives pourraient-elles faire évoluer la situation et avoir un impact réel sur la valorisation du travail en restauration, sur la rétention et le recrutement des employés et sur l'amélioration de l'image du tourisme québécois ? Peut-on s'inspirer de ce qui se fait dans d'autres pays?

En France, par exemple :

- Tout d'abord, un salaire minimum est prévu par la loi : rappelons que le SMIC est le salaire horaire en dessous duquel il est interdit de rémunérer un salarié et ce quelle que soit la forme de sa rémunération. Depuis le $1^{\mathrm{er}}$ janvier 2020, le SMIC horaire brut est fixé à 10,15 euros, soit 1159,42 euros bruts mensuellement sur la base de la durée légale hebdomadaire de 35 heures ${ }^{11}$.

- Les frais de service (10 à $15 \%$ ) sont généralement obligatoires et inscrits sur la facture du client de manière distincte, tout en étant inclus dans la plupart des cas dans le prix affiché au client.

- Perçus par le salarié ou par l'employeur, ils sont versés intégralement au personnel en contact avec la clientèle; en outre, ils entrent dans la composition même du salaire minimum ou du salaire conventionnel des personnels concernés, c'est-à-dire ceux qui sont en contact direct avec la clientèle ; si le salaire minimum ou conventionnel n'est pas atteint, 
l'employeur doit combler la différence, ce qui arrive surtout si les frais de service ou les pourboires constituent la seule rémunération du salarié.

- L'existence d'une convention sectorielle, la Convention collective nationale des hôtels, cafés, restaurants (HCR) est l'élément distinctif principal par rapport au Québec. Elle s'applique à l'ensemble des salariés du secteur (serveurs, cuisiniers, maîtres d'hôtel, etc.): selon les postes et l'ancienneté, elle fixe un salaire horaire conventionnel minimum, qui en 2019 se situe entre 10,03 euros (niveau 1, échelon 1) et 21,83 euros (niveau 5, échelon 3).

- La convention sectorielle régit également les autres conditions de travail. Il faut rappeler qu'un contrat individuel peut offrir plus que le minimum conventionnel.

\section{Éléments d'analyse}

La convention sectorielle offre une plus grande équité entre les divers personnels du secteur même si, par exemple, le personnel de cuisine ne bénéficie pas des frais de service, étant rémunéré par un salaire fixe versé par l'employeur. Le salaire conventionnel minimum est plus généreux que le SMIC. Les frais de service obligatoires, généralement inclus dans le prix affiché, n'induisent pas le client en erreur sur le prix à payer. Des patrons en France souhaitent cependant remettre en question « le service compris » afin de motiver davantage le personnel, mais cette prétention rencontre une vive opposition des syndicats qui y voient une régression sociale (Fergus, 2012).

En outre, un client satisfait de la prestation de service peut ajouter, en sus des frais de service, un petit pourboire, généralement de $5 \%$. Les touristes québécois en France auront tendance à ne pas laisser de pourboire lorsqu'ils voient que les frais de service sont inclus dans les prix affichés, de la même manière que les Français non avertis, en voyage au Québec, oublieront de laisser un pourboire car ils croient à tort que les frais de service sont inclus dans les prix affichés.

\section{En guise de conclusion sommaire}

Que faire au Québec ? Que devrait prévoir la Loi sur les normes du travail ? Pourquoi ne pas avoir une convention sectorielle qui aurait l'avantage de clarifier la situation de la rémunération et des conditions de travail pour l'ensemble du secteur? Les employeurs souhaitent davantage contrôler le partage des pourboires, ce que craignent les salariés concernés (Lahaie, 217). Ne devrait-on pas prendre le virage des frais de service inscrits obligatoirement sur la facture, voire même inscrits dans le prix affiché ? Il devrait en être de même pour les taxes.

Quel serait l'impact de l'imposition de frais de service obligatoires par opposition aux pourboires laissés à la discrétion des clients? Quel impact sur les autres conditions de travail ? Et sur l'image du tourisme québécois? Sur ces questions, des recherches plus approfondies sont nécessaires.

Des changements législatifs et réglementaires en la matière ne sont pas neutres et peuvent avoir des conséquences positives sur la valorisation des emplois, le recrutement et la rétention de la main-d'œuvre. En même temps, la nature et l'intensité du rapport de forces entre les parties prenantes du secteur peuvent retarder ces changements... 


\section{BIBLIOGRAPHIE}

\section{Monographies}

Bernard CUBERTAFOND, La création du droit, Ellipses, 1999.

Louis JOLIN, « La double interaction entre droit et tourisme », dans Lucie K. MORISSET, Bruno SARRASIN et Guillaume ÉTHIER (dir.), Épistémologie des études touristiques, PUQ, 2012.

Hans KELSEN, Théorie pure du droit, traduction de C. Esenmann, Sirey, 1962.

René LAPERRIÈRE, Les systèmes juridiques de détermination des salaires. Expériences québécoises, canadiennes et étrangères, thèse de doctorat, Montréal, tome 1, 1972.

Michel TROPPER, « Le positivisme juridique », dans Michel TROPPER (dir.), Pour une théorique juridique de l'État, PUF, 1994.

\section{Revues, journaux, sites web}

Lise BINET, « La thématique des transformations du droit et le paradigme du droit phénomène social », Les Cahiers du droit, vol. 31, 1990. 
Dominique CAMBRon-OUelLET, « Le salaire minimum sera un enjeu électoral », Métro, 4 janvier 2018. FERGUS, « Service compris : la duplicité des restaurateurs », Agora Vox, 22 novembre 2012 [https:// www.agoravox.fr/tribune-libre/article/service-compris-la-duplicite-des-126295].

Richard LAHAIE, « Pourboires : des patrons veulent mettre la main sur les revenus des serveurs », L'Aut'Journal, 27 septembre 2017.

Michel MINET, « Droit du travail : la hiérarchie des normes est-elle inversée ? », La Tribune, 29

septembre 2017 [https://www.latribune.fr/opinions/tribunes/droit-du-travail-la-hierarchie-desnormes-est-elle-inversee-752010.html].

Jean-Frédéric MOREAU et David RÉMILLARD, « Illégal, le pourboire sur la facture », Le Soleil, 26 août 2017.

\section{NOTES}

1. Extrait de l'appel à communication pour les Rendez-vous Champlain 2018.

2. Selon le Conseil québécois des ressources humaines en tourisme (CQRHT): «À l'instar de d'autres secteurs d'activité économique, l'industrie touristique connaît de plus en plus de problèmes de recrutement pour le travail saisonnier ou à temps partiel. Ceci s'explique en partie par le fait qu'une forte proportion de ces emplois est traditionnellement occupée par des jeunes étudiants dont l'effectif est actuellement en diminution. De plus, l'arrimage de l'emploi avec le calendrier scolaire pose certains défis aux gestionnaires. Enfin, dans un contexte où la population vieillit et où son taux de croissance diminue, la main-d'œuvre se fait donc de plus en plus rare et difficile à trouver, particulièrement pour des emplois à temps partiel ou saisonniers. »

En outre, selon une étude concernant le tourisme et la main-d'œuvre, $54 \%$ des emplois en tourisme en 2012 étaient dans le secteur de la restauration et 11 \% dans celui de l'hébergement. Cependant, le salaire moyen dans l'industrie du tourisme est de 37979 \$ pour les employés à temps complet, comparativement à $46656 \$$ pour l'ensemble du Québec. Dans le secteur spécifique de la restauration, il est de 31803 \$ pour les employés à temps complet. Ces données proviennent du site du CQRHT, consulté en février 2019: https://cqrht.qc.ca/portrait-delindustrie-touristique/donnees-sur-lindustrie-touristiquetouristique/.

3. Il s'agit principalement, pour le secteur du tourisme, des fonctionnaires et autres employés du gouvernement fédéral (ministères intervenant dans le secteur, la Commission canadienne du tourisme, Parcs Canada...) et des travailleurs du transport aérien, de Via Rail et, plus globalement, œuvrant dans le transport interprovincial ou international.

4. En 2017, le taux de syndicalisation au Québec dans le secteur de l'hébergement et de la restauration était de 10,3\%, le taux global pour l'ensemble des secteurs étant de 38,4\%. Source : Institut de la statistique du Québec, Taux de présence syndicale, résultats selon le sexe pour diverses caractéristiques de la main-d'œuvre et de l'emploi, Québec, Ontario, Canada, 2018.

5. Le salaire minimum interprofessionnel de croissance (SMIC) est le salaire minimum horaire en dessous duquel un salarié de plus de 18 ans ne peut être payé en France.

6. Étendue par arrêté du 3 décembre 1997, JORF du 6 décembre 1997. Dernière version publiée le 29 janvier 2018 et en vigueur le $1^{\mathrm{er}}$ avril 2018. Grille des salaires mise à jour en 2019 suite à l'augmentation du SMIC.

7. Étendue par arrêté du 2 février 1984, JONC du 17 février 1984. Dernière version publiée le 22 juin 2017, en vigueur le $1^{\mathrm{er}}$ septembre 2017. Grille des salaires mise à jour en 2019 suite à l'augmentation du SMIC.

8. Au $1^{\mathrm{er}}$ mai 2018 , le salaire minimum général était de $12,00 \$$ de l'heure et de $9,80 \$$ pour les employés à pourboire. 
9. Les exceptions sont prévues aux articles 42.1 à 42.15 .

10. L'Association des restaurateurs du Québec a rappelé qu'il est illégal d'ajouter les frais de service sans l'accord préalable du client. Ce rappel fait suite à une plainte faite par un client à qui le restaurateur avait chargé d'office $15 \%$ de frais de service sans son consentement. Le restaurateur s'est défendu en prétextant que c'était la coutume à l'égard des touristes asiatiques - or, malgré la ressemblance, le client n'en n'était pas un (Moreau et Rémillard, 2017).

11. Au $1^{\text {er }}$ janvier 2018 , le SMIC horaire brut était à 9,88 euros, soit 1498,47 euros bruts mensuellement sur la base d'une durée légale hebdomadaire de 35 heures.

12. Articles 8 et 11 de La Loi modifiant la Loi sur les normes du travail et d'autres dispositions législatives afin principalement de faciliter la conciliation famille-travail, LQ 2018, c. 21.

Voir le site web de l'Association des restaurateurs du Québec: www.Restaurateurs.ca/nouvelles (consulté le 19 juin 2018).

\section{RÉSUMÉS}

Le droit est producteur de transformations sociales. Le cadre juridique des employés de service du secteur de la restauration peut avoir un impact positif ou négatif sur le recrutement et la rétention des travailleurs. Le présent article met l'accent sur l'encadrement juridique des salaires au Québec, principalement ceux des employés à pourboire, tout en faisant un parallèle avec l'encadrement juridique en France pour mieux saisir les spécificités propres à chaque société et pour s'inspirer éventuellement du dispositif mis en place dans la société française. Des recherches sur les effets des modifications éventuelles sont à venir.

Law produces social transformations. The legal framework for service employees in the catering sector can have a positive or negative impact on the recruitment and retention of workers. This article focuses on the legal framework of wages in Quebec, mainly those of tip employees, while drawing a parallel with the legal framework in France to better understand the specifics of each country and possibly draw inspiration from the system in place in French society. Research on the effects of possible changes is to come.

\section{INDEX}

Mots-clés : restauration, emploi, tourisme, cadre juridique

Keywords : catering, employment, tourism, legal framework

\section{AUTEUR}

\section{LOUIS JOLIN}

Professeur titulaire à la retraite, actuellement professeur associé au département d'études urbaines et touristiques ESG UQAM

Domaine de spécialisation : droit du tourisme

Jolin.louis@uqam.ca 